\title{
Strong Comparison Principles for Some Nonlinear Degenerate Elliptic Equations
}

\author{
YanYan Li * Bo Wang ${ }^{\dagger}$ \\ Dedicated to the memory of Xiaqi Ding
}

\begin{abstract}
In this paper, we obtain the strong comparison principle and Hopf Lemma for locally Lipschitz viscosity solutions to a class of nonlinear degenerate elliptic operators of the form $\nabla^{2} \psi+L(x, \nabla \psi)$, including the conformal hessian operator.
\end{abstract}

Key words: Hopf Lemma; Strong Comparison Principle; Degenerate Ellipticity; Conformal invariance.

MSC2010: 35J60 35J70 35B51 35B65 35D40 53C21 58J70.

\section{Introduction}

In this paper, we establish the strong comparison principle and Hopf Lemma for locally Lipschitz viscosity solutions to a class of nonlinear degenerate elliptic operators.

For a positive integer $n \geq 2$, let $\Omega$ be an open connected bounded subset of $\mathbb{R}^{n}$, the $n$-dimensional euclidean space. For any $C^{2}$ function $u$ in $\Omega$, we consider a symmetric matrix function

$$
F[u]:=\nabla^{2} u+L(\cdot, \nabla u)
$$

where $L \in C_{l o c}^{0,1}\left(\Omega \times \mathbb{R}^{n}\right)$, is in $\mathcal{S}^{n \times n}$, the set of all $n \times n$ real symmetric matrices.

*Department of Mathematics, Rutgers University, 110 Frelinghuysen Rd, Piscataway, NJ 08854, USA. Email: yyli@math.rutgers.edu.

${ }^{\dagger}$ Corresponding author. School of Mathematics and Statistics, Beijing Institute of Technology, Beijing 100081, China. Email: wangbo89630@bit.edu.cn. 
One such matrix operator is the conformal hessian operator (see e.g. [21], 27] and the references therein), that is,

$$
A[u]=\nabla^{2} u+\nabla u \otimes \nabla u-\frac{1}{2}|\nabla u|^{2} I,
$$

where $I$ denotes the $n \times n$ identity matrix, and for $p, q \in \mathbb{R}^{n}, p \otimes q$ denotes the $n \times n$ matrix with entries $(p \otimes q)_{i j}=p_{i} q_{j}, i, j=1, \cdots, n$. Some comparison principles for this matrix operator have been studied in [22]-[25]. Comparison principles for other classes of (degenerate) elliptic operators are available in the literature. See [1]-[5], [7]-[20], [26] and the references therein.

Let $U$ be an open subset of $\mathcal{S}^{n \times n}$, satisfying

$$
0 \in \partial U, \quad U+\mathcal{P} \subset U, \quad t U \subset U, \forall t>0,
$$

where $\mathcal{P}$ is the set of all non-negative matrices. Furthermore, in order to conclude that the strong comparison principle holds, we assume Condition $U_{\nu}$, as introduced in [25], for some unit vector $\nu$ in $\mathbb{R}^{n}$ : there exists $\mu=\mu(\nu)>0$ such that

$$
U+C_{\mu}(\nu) \subset U \text {. }
$$

Here $C_{\mu}(\nu):=\left\{t(\nu \otimes \nu+A): A \in \mathcal{S}^{n \times n},\|A\|<\mu, t>0\right\}$. Some counter examples for the strong maximum principle were given in [25] to show that the condition (33) cannot be simply dropped.

Remark 1.1. If U satisfies (2),

$$
\operatorname{diag}\{1,0, \cdots, 0\} \in U
$$

and

$$
O^{t} U O \subset U, \quad \forall O \in O(n),
$$

where $O(n)$ denotes the set of $n \times n$ orthogonal matrices, then it is easy to see that $U$ satisfies (3).

Let $u, v \in C_{l o c}^{0,1}(\Omega)$. We say that

$$
F[u] \in \mathcal{S}^{n \times n} \backslash U \quad(F[v] \in \bar{U}), \quad \text { in } \Omega
$$

in the viscosity sense, if for any $x_{0} \in \Omega, \varphi \in C^{2}(\Omega),(\varphi-u)\left(x_{0}\right)=0\left((\varphi-v)\left(x_{0}\right)=0\right)$ and

$$
u-\varphi \geq 0 \quad(v-\varphi \leq 0), \quad \text { near } x_{0},
$$

there holds

$$
F[\varphi]\left(x_{0}\right) \in \mathcal{S}^{n \times n} \backslash U \quad\left(F[\varphi]\left(x_{0}\right) \in \bar{U}\right) .
$$

We have the following strong comparison principle and Hopf Lemma. 
Theorem 1.2. (Strong Comparison Principle) Let $\Omega$ be an open connected subset of $\mathbb{R}^{n}, n \geq 2, U$ be an open subset of $\mathcal{S}^{n \times n}$, satisfying (2) and Condition $U_{\nu}$ for every unit vector $\nu$ in $\mathbb{R}^{n}$, and $F$ be of the form (1) with $L \in C_{\text {loc }}^{0,1}\left(\Omega \times \mathbb{R}^{n}\right)$. Assume that $u, v \in C_{\text {loc }}^{0,1}(\Omega)$ satisfy (4) in the viscosity sense, $u \geq v$ in $\Omega$. Then either $u>v$ in $\Omega$ or $u \equiv v$ in $\Omega$.

Theorem 1.3. (Hopf Lemma) Let $\Omega$ be an open connected subset of $\mathbb{R}^{n}, n \geq 2$, $\partial \Omega$ be $C^{2}$ near a point $\hat{x} \in \partial \Omega$, and $U$ be an open subset of $\mathcal{S}^{n \times n}$, satisfying (2) and Condition $U_{\nu}$ for $\nu=\nu(\hat{x})$, the interior unit normal of $\partial \Omega$ at $\hat{x}$, and $F$ be of the form (11) with $L \in C_{l o c}^{0,1}\left(\Omega \times \mathbb{R}^{n}\right)$. Assume that $u, v \in C_{l o c}^{0,1}(\Omega \cup\{\hat{x}\})$ satisfy (4) in the viscosity sense, $u>v$ in $\Omega$ and $u(\hat{x})=v(\hat{x})$. Then we have

$$
\liminf _{s \rightarrow 0^{+}} \frac{(u-v)(\hat{x}+s \nu(\hat{x}))}{s}>0 .
$$

Remark 1.4. If $u$ and $v \in C^{2}$, then Theorems 1.2 and 1.3 were proved in [25].

\section{Proof of Theorem 1.2}

Proof of Theorem 1.2. We argue by contradiction. Suppose the conclusion is false. Since $u-v \in C_{l o c}^{0,1}(\Omega)$ is non-negative, the set $\{x \in \Omega: u=v\}$ is closed. Then there exists an open ball $B\left(x_{0}, R\right) \subset \subset \Omega$ centered at $x_{0} \in \Omega$ with radius $R>0$ such that

$$
\left\{\begin{array}{l}
u-v>0, \quad \text { in } \overline{B\left(x_{0}, R\right)} \backslash\{\hat{x}\} \\
u(\hat{x})-v(\hat{x})=0, \quad \hat{x} \in \partial B\left(x_{0}, R\right) .
\end{array}\right.
$$

We make use of the standard comparison function

$$
h(x):=e^{-\alpha\left|x-x_{0}\right|^{2}}-e^{-\alpha R^{2}}, \quad \forall \alpha>0, x \in \Omega .
$$

For $i, j=1, \cdots, n$, we have

$$
h_{i}(x)=\frac{\partial}{\partial x_{i}} h(x)=-2 \alpha\left(x_{i}-\left(x_{0}\right)_{i}\right) e^{-\alpha\left|x-x_{0}\right|^{2}},
$$

and

$$
h_{i j}(x)=\frac{\partial^{2}}{\partial x_{i} \partial x_{j}} h(x)=4 \alpha^{2} e^{-\alpha\left|x-x_{0}\right|^{2}}\left[\left(x_{i}-\left(x_{0}\right)_{i}\right)\left(x_{j}-\left(x_{0}\right)_{j}\right)-\frac{1}{2 \alpha} \delta_{i j}\right] .
$$


Choose $0<R^{\prime}<\frac{R}{2}$ such that $B\left(\hat{x}, R^{\prime}\right) \subset \subset \Omega$. For any $\delta \in\left(0, R^{\prime}\right)$, we have that for any $x \in \overline{B(\hat{x}, \delta)}$,

$$
-1 \leq h(x) \leq 1, \quad|\nabla h(x)|+\left|\nabla^{2} h(x)\right| \leq C
$$

for some $C>0$ independent of $\delta$ and $\alpha$.

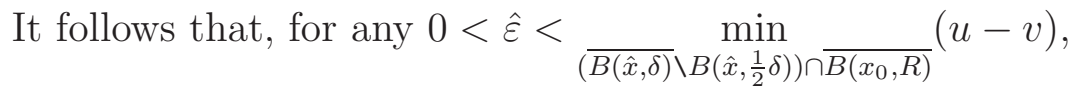

$$
u-v-\hat{\varepsilon} h>0, \quad \text { on } \overline{B(\hat{x}, \delta)} \backslash B\left(\hat{x}, \frac{1}{2} \delta\right), \quad(u-v-\hat{\varepsilon} h)(\hat{x})=0 .
$$

Indeed, by (8) and the fact that $h<0$ outside $\overline{B\left(x_{0}, R\right)}$, for any $x \in\left(\overline{B(\hat{x}, \delta)} \backslash B\left(\hat{x}, \frac{1}{2} \delta\right)\right) \backslash \overline{B\left(x_{0}, R\right)}$,

$$
(u-v)(x) \geq 0>\hat{\varepsilon} h(x)
$$

and for any $x \in\left(\overline{B(\hat{x}, \delta)} \backslash B\left(\hat{x}, \frac{1}{2} \delta\right)\right) \cap \overline{B\left(x_{0}, R\right)}$,

$$
(u-v)(x) \geq \min _{\left(\overline{B(\hat{x}, \delta)} \backslash B\left(\hat{x}, \frac{1}{2} \delta\right)\right) \cap \overline{B\left(x_{0}, R\right)}}(u-v)>\hat{\varepsilon} \geq \hat{\varepsilon} h(x) .
$$

For any $\epsilon>0$, we define the $\epsilon$-lower and upper envelope of $u$ and $v$ as

$$
u_{\epsilon}(x):=\min _{y \in \overline{B\left(x_{0}, R\right)} \cup \overline{B\left(\hat{x}, R^{\prime}\right)}}\left\{u(y)+\frac{1}{\epsilon}|x-y|^{2}\right\}, \quad \forall x \in \overline{B\left(x_{0}, R\right)} \cup \overline{B\left(\hat{x}, R^{\prime}\right)},
$$

and

$$
v^{\epsilon}(x):=\max _{y \in \overline{B\left(x_{0}, R\right)} \cup \overline{B\left(\hat{x}, R^{\prime}\right)}}\left\{v(y)-\frac{1}{\epsilon}|x-y|^{2}\right\}, \quad \forall x \in \overline{B\left(x_{0}, R\right)} \cup \overline{B\left(\hat{x}, R^{\prime}\right)},
$$

respectively.

Then we conclude that there exists $\epsilon_{0}=\epsilon_{0}(\delta, \alpha, \hat{\varepsilon})$ such that for $0<\epsilon<\epsilon_{0}$,

$$
\frac{\min }{B(\hat{x}, \delta)}\left(u_{\epsilon}-v^{\epsilon}-\hat{\varepsilon} h\right) \leq 0, \quad u_{\epsilon}-v^{\epsilon}-\hat{\varepsilon} h>0 \text { on } \overline{B(\hat{x}, \delta)} \backslash B\left(\hat{x}, \frac{1}{2} \delta\right) .
$$

Indeed, the first part of (10) follows from the definitions of $u_{\epsilon}$ and $v^{\epsilon}$, and the fact that $h(\hat{x})=0 ;\left(u_{\epsilon}-v^{\epsilon}-\hat{\varepsilon} h\right)(\hat{x}) \leq(u-v)(\hat{x})=0$. Now we prove the second part of (10). By theorem 5.1 (a) in [6], we have that

$$
u_{\epsilon}-v^{\epsilon} \uparrow u-v \quad \text { uniformly on } B\left(x_{0}, R\right) \cup B\left(\hat{x}, R^{\prime}\right), \quad \text { as } \epsilon \rightarrow 0 \text {. }
$$


It follows that for any $M>0$, there exists $\epsilon_{0}(M)>0$ such that

$$
\left(u_{\epsilon}-v^{\epsilon}-\hat{\varepsilon} h\right)(x)>\frac{\min }{B(\hat{x}, \delta) \backslash B\left(\hat{x}, \frac{1}{2} \delta\right)}(u-v-\hat{\varepsilon} h)-M
$$

for any $0<\epsilon<\epsilon_{0}$ and any $x \in \overline{B(\hat{x}, \delta)} \backslash B\left(\hat{x}, \frac{1}{2} \delta\right)$. Then by taking $0<M<$ $\frac{1}{2} \frac{\min _{B(\hat{x}, \delta) \backslash B\left(\hat{x}, \frac{1}{2} \delta\right)}}{}(u-v-\hat{\varepsilon} h),(10)$ is obtained.

It follows from (10) that there exists $\bar{\eta}=\bar{\eta}(\delta, \alpha, \hat{\varepsilon})>0$ such that for any $\eta \in$ $(0, \bar{\eta})$, there exists $\tau=\tau(\epsilon, \eta, \delta, \alpha, \hat{\varepsilon}) \in \mathbb{R}^{1}$ such that

$$
\frac{\min }{B(\hat{x}, \delta)}\left(u_{\epsilon}-v^{\epsilon}-\hat{\varepsilon} h-\tau\right)=-\eta, \quad u_{\epsilon}-v^{\epsilon}-\hat{\varepsilon} h-\tau>0 \text { on } \overline{B(\hat{x}, \delta)} \backslash B\left(\hat{x}, \frac{1}{2} \delta\right) .
$$

Let

$$
\xi_{\epsilon}:=u_{\epsilon}-v^{\epsilon}-\hat{\varepsilon} h-\tau,
$$

and $\Gamma_{\xi_{\epsilon}^{-}}$denote the convex envelope of $\xi_{\epsilon}^{-}:=-\min \left\{\xi_{\epsilon}, 0\right\}$ on $\overline{B(\hat{x}, \delta)}$. Then by $(20)$ in [24] and (8), we have

$$
\nabla^{2} \xi_{\epsilon} \leq \frac{4}{\epsilon} I+C \hat{\varepsilon} I \quad \text { a.e. in } B\left(\hat{x}, \frac{1}{2} \delta\right) .
$$

And by lemma 3.5 in [6], we have

$$
\int_{\left\{\xi_{\epsilon}=\Gamma_{\xi_{\epsilon}^{-}}\right\}} \operatorname{det}\left(\nabla^{2} \Gamma_{\xi_{\epsilon}^{-}}\right)>0,
$$

which implies that the Lebesgue measure of $\left\{\xi_{\epsilon}=\Gamma_{\xi_{\epsilon}^{-}}\right\}$is positive. Then there exists $x_{\epsilon, \eta} \in\left\{\xi_{\epsilon}=\Gamma_{\xi_{\epsilon}^{-}}\right\} \cap B\left(\hat{x}, \frac{1}{2} \delta\right)$ such that both of $v^{\epsilon}$ and $u_{\epsilon}$ are punctually second order differentiable at $x_{\epsilon, \eta}$,

$$
\begin{gathered}
0>\xi_{\epsilon}\left(x_{\epsilon, \eta}\right) \geq-\eta \\
\left|\nabla \xi_{\epsilon}\left(x_{\epsilon, \eta}\right)\right| \leq C \eta,
\end{gathered}
$$

and

$$
\nabla^{2} \xi_{\epsilon}\left(x_{\epsilon, \eta}\right)=\nabla^{2}\left(u_{\epsilon}-v^{\epsilon}-\hat{\varepsilon} h\right)\left(x_{\epsilon, \eta}\right) \geq 0 .
$$

For $x_{\epsilon, \eta} \in \Omega$, by the definitions of $u_{\epsilon}$ and $v^{\epsilon}$, there exist $\left(x_{\epsilon, \eta}\right)_{*}$ and $\left(x_{\epsilon, \eta}\right)^{*} \in \Omega$ such that

$$
u_{\epsilon}\left(x_{\epsilon, \eta}\right)=u\left(\left(x_{\epsilon, \eta}\right)_{*}\right)+\frac{1}{\epsilon}\left|\left(x_{\epsilon, \eta}\right)_{*}-x_{\epsilon, \eta}\right|^{2},
$$

and

$$
v^{\epsilon}\left(x_{\epsilon, \eta}\right)=v\left(\left(x_{\epsilon, \eta}\right)^{*}\right)-\frac{1}{\epsilon}\left|\left(x_{\epsilon, \eta}\right)^{*}-x_{\epsilon, \eta}\right|^{2} .
$$


Since $u$ and $v \in C_{l o c}^{0,1}(\Omega)$, by (2.6) and (2.7) in [23], we have

$$
\left|\left(x_{\epsilon, \eta}\right)_{*}-x_{\epsilon, \eta}\right|+\left|\left(x_{\epsilon, \eta}\right)^{*}-x_{\epsilon, \eta}\right| \leq C_{1} \epsilon,
$$

and

$$
\left|\nabla u_{\epsilon}\left(x_{\epsilon, \eta}\right)\right|+\left|\nabla v^{\epsilon}\left(x_{\epsilon, \eta}\right)\right| \leq C_{2},
$$

where $C_{1}$ and $C_{2}$ are two universal positive constant independent of $\epsilon$ and $\eta$.

Since $u_{\epsilon}$ is punctually second order differentiable at $x_{\epsilon, \eta}$, we have

$$
u_{\epsilon}\left(x_{\epsilon, \eta}+z\right) \geq u_{\epsilon}\left(x_{\epsilon, \eta}\right)+\nabla u_{\epsilon}\left(x_{\epsilon, \eta}\right) \cdot z+\frac{1}{2} z^{T} \nabla^{2} u_{\epsilon}\left(x_{\epsilon, \eta}\right) z+o\left(|z|^{2}\right), \quad \text { as } z \rightarrow 0
$$

By the definition of $u_{\epsilon}$, we have

$$
u_{\epsilon}\left(x_{\epsilon, \eta}+z\right) \leq u\left(\left(x_{\epsilon, \eta}\right)_{*}+z\right)+\frac{1}{\epsilon}\left|\left(x_{\epsilon, \eta}\right)_{*}-x_{\epsilon, \eta}\right|^{2},
$$

and therefore, in view of (17),

$$
\begin{aligned}
u\left(\left(x_{\epsilon, \eta}\right)_{*}+z\right) & \geq u_{\epsilon}\left(x_{\epsilon, \eta}+z\right)-\frac{1}{\epsilon}\left|\left(x_{\epsilon, \eta}\right)_{*}-x_{\epsilon, \eta}\right|^{2} \\
& \geq P_{\epsilon}\left(\left(x_{\epsilon, \eta}\right)_{*}+z\right)+o\left(|z|^{2}\right), \quad \text { as } z \rightarrow 0,
\end{aligned}
$$

where $P_{\epsilon}$ is a quadratic polynomial with

$$
\begin{aligned}
P_{\epsilon}\left(\left(x_{\epsilon, \eta}\right)_{*}\right) & \left.=u_{\epsilon}\left(x_{\epsilon, \eta}\right)-\frac{1}{\epsilon}\left|\left(x_{\epsilon, \eta}\right)_{*}-x_{\epsilon, \eta}\right|^{2}=u\left(x_{\epsilon, \eta}\right)_{*}\right), \\
\nabla P_{\epsilon}\left(\left(x_{\epsilon, \eta}\right)_{*}\right) & =\nabla u_{\epsilon}\left(x_{\epsilon, \eta}\right), \\
\nabla^{2} P_{\epsilon}\left(\left(x_{\epsilon, \eta}\right)_{*}\right) & =\nabla^{2} u_{\epsilon}\left(x_{\epsilon, \eta}\right) .
\end{aligned}
$$

Since $u$ satisfies (41) in the viscosity sense, we thus have

$$
\nabla^{2} u_{\epsilon}\left(x_{\epsilon, \eta}\right)+L\left(\left(x_{\epsilon, \eta}\right)_{*}, \nabla u_{\epsilon}\left(x_{\epsilon, \eta}\right)\right)=F\left[P_{\epsilon}\right]\left(\left(x_{\epsilon, \eta}\right)_{*}\right) \in \mathcal{S}^{n \times n} \backslash U
$$

On the other hand, in view of $(\underline{15}),(\underline{16})$ and the fact that $L \in C_{l o c}^{0,1}\left(\Omega \times \mathbb{R}^{n}\right)$,

$$
L\left(x_{\epsilon, \eta}, \nabla u_{\epsilon}\left(x_{\epsilon, \eta}\right)\right)-L\left(\left(x_{\epsilon, \eta}\right)_{*}, \nabla u_{\epsilon}\left(x_{\epsilon, \eta}\right)\right) \leq C\left|x_{\epsilon, \eta}-\left(x_{\epsilon, \eta}\right)_{*}\right| I \leq a_{1} \epsilon I,
$$

where $C$ and $a_{1}>0$ are universal constants.

It follows from (2), (18) and (19) that

$$
F\left[u_{\epsilon}\right]\left(x_{\epsilon, \eta}\right)-a_{1} \epsilon I \in \mathcal{S}^{n \times n} \backslash U .
$$


Analogusly, we can obtain

$$
F\left[v^{\epsilon}\right]\left(x_{\epsilon, \eta}\right)+a_{2} \epsilon I \in \bar{U}
$$

for some universal constants $a_{2}>0$.

By (13), (14), (16) and the fact that $L \in C_{l o c}^{0,1}\left(\Omega \times \mathbb{R}^{n}\right)$,

$$
\begin{aligned}
F\left[u_{\epsilon}\right]\left(x_{\epsilon, \eta}\right) & \geq \nabla^{2}\left(v^{\epsilon}+\hat{\varepsilon} h\right)\left(x_{\epsilon, \eta}\right)+L\left(x_{\epsilon, \eta}, \nabla u_{\epsilon}\left(x_{\epsilon, \eta}\right)\right) \\
& =F\left[v^{\epsilon}+\hat{\varepsilon} h\right]\left(x_{\epsilon, \eta}\right)+L\left(x_{\epsilon, \eta}, \nabla u_{\epsilon}\left(x_{\epsilon, \eta}\right)\right)-L\left(x_{\epsilon, \eta}, \nabla v^{\epsilon}\left(x_{\epsilon, \eta}\right)\right) \\
& \left.\geq F\left[v^{\epsilon}+\hat{\varepsilon} h\right]\left(x_{\epsilon, \eta}\right)-C \mid \nabla\left(u_{\epsilon}-v^{\epsilon}\right)\left(x_{\epsilon, \eta}\right)\right) \\
& \geq F\left[v^{\epsilon}+\hat{\varepsilon} h\right]\left(x_{\epsilon, \eta}\right)-C\left(\eta+\hat{\varepsilon}\left|\nabla h\left(x_{\epsilon, \eta}\right)\right|\right) I .
\end{aligned}
$$

By (8), (16) and the fact that $L \in C_{l o c}^{0,1}\left(\Omega \times \mathbb{R}^{n}\right)$, we have

$$
\begin{aligned}
& F\left[v^{\epsilon}+\hat{\varepsilon} h\right]\left(x_{\epsilon, \eta}\right) \\
= & F\left[v^{\epsilon}\right]\left(x_{\epsilon, \eta}\right)+\hat{\varepsilon} \nabla^{2} h\left(x_{\epsilon, \eta}\right)+L\left(x_{\epsilon, \eta}, \nabla\left(v^{\epsilon}+\hat{\varepsilon} h\right)\left(x_{\epsilon, \eta}\right)\right)-L\left(x_{\epsilon, \eta}, \nabla v^{\epsilon}\left(x_{\epsilon, \eta}\right)\right) \\
\geq & F\left[v^{\epsilon}\right]\left(x_{\epsilon, \eta}\right)+\hat{\varepsilon}\left[\nabla^{2} h\left(x_{\epsilon, \eta}\right)-C\left|\nabla h\left(x_{\epsilon, \eta}\right)\right| I\right] .
\end{aligned}
$$

Then by (6), (17) and the fact $\left|x_{\epsilon, \eta}-x_{0}\right|<2 R$,

$$
\begin{aligned}
& \nabla^{2} h\left(x_{\epsilon, \eta}\right)-C\left|\nabla h\left(x_{\epsilon, \eta}\right)\right| I \\
= & 4 \alpha^{2} e^{-\alpha\left|x_{\epsilon, \eta}-x_{0}\right|^{2}}\left[\left(x_{\epsilon, \eta}-x_{0}\right) \otimes\left(x_{\epsilon, \eta}-x_{0}\right)-\frac{1}{2 \alpha} I-\frac{C}{4 \alpha}\left|x_{\epsilon, \eta}-x_{0}\right| I\right] \\
\geq & 4 \alpha^{2} e^{-\alpha\left|x_{\epsilon, \eta}-x_{0}\right|^{2}}\left[\left(x_{\epsilon, \eta}-x_{0}\right) \otimes\left(x_{\epsilon, \eta}-x_{0}\right)-\frac{C}{\alpha} I\right] \\
\geq & 4 \alpha^{2} e^{-\alpha\left|x_{\epsilon, \eta}-x_{0}\right|^{2}}\left[\left(\hat{x}-x_{0}\right) \otimes\left(\hat{x}-x_{0}\right)-C \delta R I-\frac{C}{\alpha} I\right] \\
= & 4 R^{2} \alpha^{2} e^{-\alpha\left|x_{\epsilon, \eta}-x_{0}\right|^{2}}\left[\left(\frac{\hat{x}-x_{0}}{R}\right) \otimes\left(\frac{\hat{x}-x_{0}}{R}\right)-C \delta I-\frac{C}{\alpha} I\right] \\
\geq & 4 R^{2} \alpha^{2} e^{-4 R^{2} \alpha}\left[\left(\frac{\hat{x}-x_{0}}{R}\right) \otimes\left(\frac{\hat{x}-x_{0}}{R}\right)-C \delta I-\frac{C}{\alpha} I\right] .
\end{aligned}
$$

Inserting (23) into (22), we have

$$
F\left[v^{\epsilon}+\varepsilon h\right]\left(x_{\epsilon, \eta}\right) \geq F\left[v^{\epsilon}\right]\left(x_{\epsilon, \eta}\right)+4 R^{2} \hat{\varepsilon} \alpha^{2} e^{-4 R^{2} \alpha}\left[\left(\frac{\hat{x}-x_{0}}{R}\right) \otimes\left(\frac{\hat{x}-x_{0}}{R}\right)-C \delta I-\frac{C}{\alpha} I\right] .
$$


It follows from (21) and (24) that

$$
\begin{aligned}
& \quad F\left[u_{\epsilon}\right]\left(x_{\epsilon, \eta}\right)-a_{1} \epsilon I \\
& \geq F\left[v^{\epsilon}\right]\left(x_{\epsilon, \eta}\right)+a_{2} \epsilon I \\
& \quad+4 R^{2} \hat{\varepsilon} \alpha^{2} e^{-4 R^{2} \alpha}\left[\left(\frac{\hat{x}-x_{0}}{R}\right) \otimes\left(\frac{\hat{x}-x_{0}}{R}\right)-C \delta I-\frac{C}{\alpha} I-C \frac{e^{4 R^{2} \alpha}}{\hat{\varepsilon} \alpha^{2}}(\epsilon+\eta) I\right] .
\end{aligned}
$$

We can firstly fix the value of small $\delta>0$ and a large $\alpha>1$, then fix the value of small $\hat{\varepsilon}>0$, and lastly fix the value of small $\epsilon$ and $\eta>0$ such that

$$
\left\|C \delta I+\frac{C}{\alpha} I+C \frac{e^{4 R^{2} \alpha}}{\hat{\varepsilon} \alpha^{2}}(\epsilon+\eta) I\right\|<\frac{1}{2} \mu\left(\frac{\hat{x}-x_{0}}{R}\right),
$$

where $\mu$ is obtained from condition (3).

Therefore, by (3) and (25), we have that

$$
F\left[u_{\epsilon}\right]\left(x_{\epsilon, \eta}\right)-a_{1} \epsilon I \in U,
$$

which is a contradiction with (20). Theorem 1.2 is proved.

\section{Proof of Theorem 1.3}

Proof of Theorem 1.3. Since $\partial \Omega$ is $C^{2}$ near $\hat{x}$, there exists an open ball $B\left(x_{0}, R\right) \subset \Omega$ such that $\overline{B\left(x_{0}, R\right)} \cap \partial \Omega=\{\hat{x}\}$ and

$$
\left\{\begin{array}{l}
u-v>0, \quad \text { in } \overline{B\left(x_{0}, R\right)} \backslash\{\hat{x}\} \\
u(\hat{x})-v(\hat{x})=0
\end{array}\right.
$$

Let $h$ be defined as in (5). We work in the domain

$$
A_{\delta}:=B(\hat{x}, \delta) \cap B\left(x_{0}, R\right) .
$$

It is easy to see that

$$
u-v \geq \hat{\varepsilon} h, \quad \text { on } \partial A_{\delta}
$$

for any $0<\delta<\frac{R}{2}$ and $0<\hat{\varepsilon}<\min _{\partial B(\hat{x}, \delta) \cap \overline{B\left(x_{0}, R\right)}}(u-v)$. 
We claim that for $\varepsilon$ small enough,

$$
u-v \geq \hat{\varepsilon} h, \quad \text { on } \overline{A_{\delta}} .
$$

Once the claim is proved, then we have that

$$
\liminf _{s \rightarrow 0^{+}} \frac{(u-v)(\hat{x}+s \nu(\hat{x}))}{s} \geq \hat{\varepsilon} \liminf _{s \rightarrow 0^{+}} \frac{h(\hat{x}+s \nu(\hat{x}))}{s}=2 \alpha R e^{-\alpha R^{2}}>0 .
$$

Therefore, in order to finish the proof of Theorem 1.3, we only need to prove the above claim. Suppose the contrary, that is,

$$
\zeta=\zeta(\hat{\varepsilon}, \alpha, \delta):=\min _{\overline{A_{\delta}}}(u-v-\hat{\varepsilon} h)<0 .
$$

It follows that

$$
\min _{\overline{A_{\delta}}}(u-v-\hat{\varepsilon} h-\zeta)=0, \quad u-v-\hat{\varepsilon} h-\zeta \geq-\zeta>0 \text { on } \partial A_{\delta} .
$$

Now we can follow the argument as in the proof of Theorem 1.2 to get a contradiction. Theorem 1.3 is proved.

Acknowledgments. Li is partially supported by NSF grant DMS-1501004. Wang is partially supported by NNSF (11701027).

\section{References}

[1] M. E. Amendola, G. Galise, And A. Vitolo, Riesz capacity, maximum principle, and removable sets of fully nonlinear second-order elliptic operators, Differential Integral Equations, 26 (2013), pp. 845-866.

[2] M. BARDi AND F. DA Lio, On the strong maximum principle for fully nonlinear degenerate elliptic equations, Arch. Math. (Basel), 73 (1999), pp. 276-285.

[3] H. Berestycki, I. Capuzzo Dolcetta, A. Porretta, And L. Rossi, Maximum principle and generalized principal eigenvalue for degenerate elliptic operators, J. Math. Pures Appl. (9), 103 (2015), pp. 1276-1293.

[4] I. Birindelli And F. Demengel, Comparison principle and Liouville type results for singular fully nonlinear operators, Ann. Fac. Sci. Toulouse Math. (6), 13 (2004), pp. 261-287. 
[5] — Eigenvalue, maximum principle and regularity for fully non linear homogeneous operators, Commun. Pure Appl. Anal., 6 (2007), pp. 335-366.

[6] L. Caffarelli And X. CABré, Fully nonlinear elliptic equations, vol. 43 of American Mathematical Society Colloquium Publications, American Mathematical Society, Providence, RI, 1995.

[7] L. Caffarelli, Y. Y. Li, And L. NirenberG, Some remarks on singular solutions of nonlinear elliptic equations III: viscosity solutions including parabolic operators, Comm. Pure Appl. Math., 66 (2013), pp. 109-143.

[8] M. G. Crandall, H. Ishit, And P.-L. Lions, User's guide to viscosity solutions of second order partial differential equations, Bull. Amer. Math. Soc. (N.S.), 27 (1992), pp. 1-67.

[9] I. C. Dolcetta And A. Vitolo, On the maximum principle for viscosity solutions of fully nonlinear elliptic equations in general domains, Matematiche (Catania), 62 (2007), pp. 69-91.

[10] — , The weak maximum principle for degenerate elliptic operators in unbounded domains, (preprint).

[11] F. R. Harvey And H. B. LAwson, JR., Existence, uniqueness and removable singularities for nonlinear partial differential equations in geometry, in Surveys in differential geometry. Geometry and topology, vol. 18 of Surv. Differ. Geom., Int. Press, Somerville, MA, 2013, pp. 103-156.

[12] _ Characterizing the strong maximum principle for constant coefficient subequations, Rend. Mat. Appl., (7) 37 (2016), no. 1-2, pp. 63-104.

[13] H. IsHII, On uniqueness and existence of viscosity solutions of fully nonlinear second-order elliptic PDEs, Comm. Pure Appl. Math., 42 (1989), pp. 15-45.

[14] H. IshiI AND P.-L. LiOns, Viscosity solutions of fully nonlinear secondorder elliptic partial differential equations, J. Differential Equations, 83 (1990), pp. 26-78.

[15] R. JENSEN, The maximum principle for viscosity solutions of fully nonlinear second order partial differential equations, Arch. Rational Mech. Anal., 101 (1988), pp. 1-27. 
[16] B. Kawohl And N. Kutev, Strong maximum principle for semicontinuous viscosity solutions of nonlinear partial differential equations, Arch. Math. (Basel), 70 (1998), pp. 470-478.

[17] — Comparison principle and Lipschitz regularity for viscosity solutions of some classes of nonlinear partial differential equations, Funkcial. Ekvac., 43 (2000), pp. 241-253.

[18] — Comparison principle for viscosity solutions of fully nonlinear, degenerate elliptic equations, Comm. Partial Differential Equations, 32 (2007), pp. 12091224 .

[19] S. KoIke And T. Kosugi, Remarks on the comparison principle for quasilinear PDE with no zeroth order terms, Commun. Pure Appl. Anal., 14 (2015), pp. 133-142.

[20] S. KOIKE AND O. LEY, Comparison principle for unbounded viscosity solutions of degenerate elliptic PDEs with gradient superlinear terms, J. Math. Anal. Appl., 381 (2011), pp. 110-120.

[21] A. Li AND Y. Y. Li, On some conformally invariant fully nonlinear equations, Comm. Pure Appl. Math., 56 (2003), pp. 1416-1464.

[22] Y. Y. LI, Degenerate conformally invariant fully nonlinear elliptic equations, Arch. Rational. Mech. and Anal. 186 (2007), pp. 25-51.

[23] _ Local gradient estimates of solutions to some conformally invariant fully nonlinear equations, Comm. Pure Appl. Math., 62 (2009), pp. 1293-1326. (C. R. Math. Acad. Sci. Paris 343 (2006), no. 4, 249-252).

[24] Y. Y. Li, L. NGuyen, And B. Wang, Comparison principles and Lipschitz regularity for some nonlinear degenerate elliptic equations, (2016). http://arxiv.org/abs/1612.09418v1. To appear in Calc. Var. Partial Differential Equations.

[25] Y. Y. Li and L. Nirenberg, A miscellany, in Percorsi incrociati (in ricordo di Vittorio Cafagna), Collana Scientifica di Ateneo, Universita di Salerno, 2010, pp. 193-208. http://arxiv.org/abs/0910.0323.

[26] N. S. Trudinger, Comparison principles and pointwise estimates for viscosity solutions of nonlinear elliptic equations, Rev. Mat. Iberoamericana, 4 (1988), pp. $453-468$. 
[27] J. ViaClovsky, Conformal geometry, contact geometry, and the calculus of variations, Duke Math. J. 101 (2000), pp. 283-316. 